\title{
Staggered repulsion of transmission eigenvalues in symmetric open mesoscopic systems
}

\author{
Marten Kopp and Henning Schomerus \\ Department of Physics, Lancaster University, Lancaster, LA1 4YB, United Kingdom \\ Stefan Rotter \\ Department of Applied Physics, Yale University, New Haven, CT 06520, USA
}

(Dated: August 8, 2008)

\begin{abstract}
Quantum systems with discrete symmetries can usually be desymmetrized, but this strategy fails when considering transport in open systems with a symmetry that maps different openings onto each other. We investigate the joint probability density of transmission eigenvalues for such systems in random-matrix theory. In the orthogonal symmetry class we show that the eigenvalue statistics manifests level repulsion between only every second transmission eigenvalue. This finds its natural statistical interpretation as a staggered superposition of two eigenvalue sequences. For a large number of channels, the statistics for a system with a lead-transposing symmetry approaches that of a superposition of two uncorrelated sets of eigenvalues as in systems with a lead-preserving symmetry (which can be desymmetrized). These predictions are confirmed by numerical computations of the transmission-eigenvalue spacing distribution for quantum billiards and for the open kicked rotator.
\end{abstract}

PACS numbers: 05.45.Mt, 05.60.Gg, 73.23.-b

\section{INTRODUCTION}

Mesoscopic systems exhibit variations in their phasecoherent electronic transport properties that are conveniently characterized via statistical approaches. Geometries that classically give rise to chaotic motion typically display universal fluctuations which can be captured using ensembles of random scattering matrices [1]. For normal conductors the universal properties fall into Dyson's three universality classes with symmetry index $\beta=1,2,4[2]$, while a further seven universality classes can be identified in the presence of superconducting or chiral particle-hole symmetries [3]. A powerful tool to distinguish these ensembles is the amount of level repulsion between the transmission eigenvalues $T_{n}$. These eigenvalues determine fundamental transport properties such as the conductance $G$ or the shot-noise Fano factor $F[1,4]$. In the Dyson ensembles, the probability density to find two closely spaced adjacent transmission eigenvalues with small distance $s=T_{n+1}-T_{n}$ is suppressed as $P(s) \propto s^{\beta}[5,6]$. This introduces a stiffness in the transmission-eigenvalue sequence which suppresses the fluctuations of the conductance and of the Fano factor when compared to the case of uncorrelated transmission eigenvalues (the latter being characteristic for classically integrable systems with a complete set of good quantum numbers) $[1,4]$.

From the investigation of closed systems it is well known that discrete symmetries result in a reduction of level repulsion. In such systems, desymmetrization delivers independent variants of the system which differ by the boundary conditions on the symmetry lines (e.g., Dirichlet and Neumann boundary conditions for eigenfunctions of odd and even parity, respectively). The statistics of the desymmetrized versions can depend on the dimensionality of the irreducible representation [7], but still remain within the conventional universality classes. The combined level statistics is then built by superimposing the independent level sequences of the desymmetrized variants [5]. In open systems, this concept of desymmetrization can be directly applied as long as the symmetry in question preserves the shape and position of the leads $[8,9]$.

This paper is motivated by the observation that systems with a lead-transposing symmetry (which maps different openings onto each other while leaving the dynamics in the system unchanged) exhibit transport properties that can only be understood as collective features of the desymmetrized variants of the system $[8,9]$. An obvious indication of this complication is the fact that the symmetry-reduced variants only possess a reduced number of leads (we concentrate on systems with two leads, for which the desymmetrized variants only possess a single lead). We demonstrate that such systems exhibit nevertheless a reduced repulsion of transmission eigenvalues which is similar to that for systems with a lead-preserving symmetry. For a large number $N$ of transport channels, the local statistical fluctuations in the eigenvalue sequence indeed become indistinguishable for both types of symmetry. However, for a small numbers of channels, the statistics differ from each other, which can be traced back to the absence or presence of $1 / N$ corrections in these ensembles.

In the specific case of $\beta=1$, we derive exact closed expressions for the joint probability density of transmission eigenvalues thereby gaining detailed insight into these statistical features. In particular, we find for both the lead-preserving and the lead-transposing symmetry class that level repulsion occurs only between every second transmission eigenvalue. The fluctuations in the transmission eigenvalue sequence hence find their most natural statistical interpretation in a staggered superposition 
of two independent level sequences. In such a superposition, the transmission eigenvalues alternate between the two sequences when they are ordered by magnitude.

The exact expressions for the joint probability density with $\beta=1$ are different for the two types of symmetry. Hence, the details of the transport statistics for a lead-transposing symmetry deviate from those for a leadpreserving symmetry. We show that these deviations are most significant for a small number of channels, while for a large number of channels the local eigenvalue statistics do indeed converge onto each other.

Previous studies of open systems with lead-transposing or lead-preserving symmetries have derived the distribution of transmission eigenvalues for one or two open channels and the one-point density for arbitrary numbers of channels [8-11]. For time-reversal symmetric systems with $\beta=1$, a key observation of these works was an enhancement of universal fluctuations for both types of symmetry (when compared to asymmetric systems). For systems with a lead-transposing symmetry it was found that the weak localization correction is vanishing, leading to ensemble averaged expressions for the conductance and for the shot noise Fano factor which are entirely independent of the channel number N [11]. The underlying staggered level statistics embodied in the joint distribution of transmission eigenvalues provides a unifying explanation for all of these observations. We verify our predictions by numerical computations for quantum billiards $[12,13]$ and for the open kicked rotator [14-16].

This paper is organized as follows. Section II provides background information on the scattering approach to transport and on standard random-matrix theory. In Sec. III we revisit the case of systems with a lead-preserving symmetry and provide the exact reformulation of the eigenvalue statistics in the orthogonal symmetry class $(\beta=1)$ as a staggered superposition of two eigenvalue sequences. Section IV concerns systems with a leadtransposing symmetry. In particular, for $\beta=1$ we derive the exact joint probability density of transmission eigenvalues for arbitrary $N$, and show that this again takes the form of a staggered eigenvalue sequence. We also describe the convergence of the local statistics for both types of symmetry, which emerges in the limit $N \rightarrow \infty$. Section $\mathrm{V}$ provides numerical results that illustrate the similarities and differences of the random-matrix ensembles for the two symmetry classes. This section also contains the comparison to specific model systems. Section VI provides a summary and discussion of our main results.

\section{BASIC CONCEPTS}

\section{A. Scattering approach to transport}

Figure 1 depicts open two-dimensional quantum billiards representing mesoscopic systems with two attached leads ( $\mathrm{L}$ - left and $\mathrm{R}$ - right), each carrying $N$ incoming and $N$ outgoing modes. The systems in Fig.
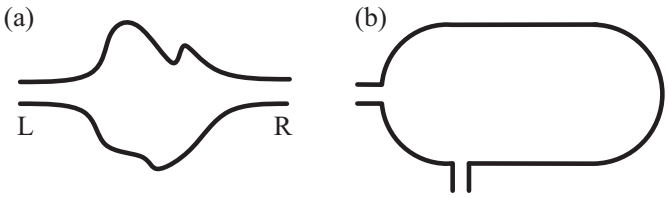

(c)

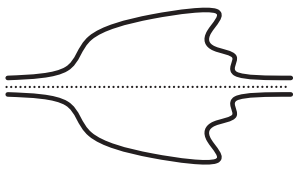

(d)

(e)

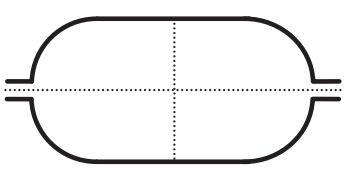

(f)
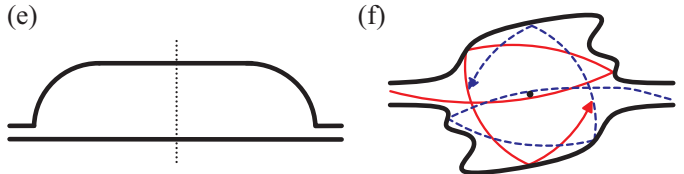

FIG. 1: (Color online) Sketches of quantum billiards (a,b) without any spatial symmetry, (c) with a lead-preserving symmetry, (d) with both a lead-preserving as well as a leadtransposing symmetry, (e) with a lead-transposing reflection symmetry, and (f) with a lead-transposing inversion symmetry. The inversion symmetry in panel (f) survives in the presence of a finite magnetic field, as is indicated by a symmetric pair of trajectories.

1(a,b) are asymmetric while those in Fig. 1(e,f) possess a lead-transposing reflection or inversion symmetry, respectively. In the middle panels, Fig. 1(c) shows a system with a lead-preserving symmetry, and Fig. 1(d) shows a system which possesses both a lead-preserving (up-down) and a lead-transposing (right-left) symmetry.

In order to describe the phase-coherent transport through these systems for small bias voltage $V$, one solves the Schrödinger equation for fixed values of the $2 N$ amplitudes $\mathbf{a}=\left[a_{n}^{(L)}, a_{n}^{(R)}\right]^{T}$ in the incoming modes. This results in linear relations $\mathbf{b}=S \mathbf{a}$ for the $2 N$ amplitudes $\mathbf{b}=\left[b_{n}^{(L)}, b_{n}^{(R)}\right]^{T}$ in the outgoing modes, which delivers a $2 N \times 2 N$-dimensional scattering matrix of the form

$$
S=\left(\begin{array}{ll}
r & t^{\prime} \\
t & r^{\prime}
\end{array}\right)
$$

Here $r, r^{\prime}, t, t^{\prime}$ are $N \times N$-dimensional matrices describing reflection at each lead and transmission from one lead to the other, respectively.

The scattering matrix is unitary, and its structure is further constrained by symmetries of the system. The three main universality classes arise for systems with time-reversal and spin-rotation symmetry (orthogonal symmetry class with $S=S^{T}$, symmetry index $\beta=1$ ), systems without time-reversal symmetry (unitary symmetry class with no constraints on $S, \beta=2$ ), and systems with time-reversal but broken spin-rotation symmetry (symplectic symmetry class composed of self-dual matrices $S=S^{R}, \beta=4$ ). Spatial symmetries entail additional constraints on the scattering matrix, which are detailed in Secs. III and IV. 
The transmission eigenvalues $T_{n}$ are defined as the eigenvalues of the hermitian matrix $t t^{\dagger}$. In the case of spin-independent transport or Kramers degeneracy (the latter occurs for $\beta=4$ ), the transmission eigenvalues are twofold degenerate. We then only account for each pair of eigenvalues once and introduce a spin-degeneracy factor $\alpha=2$. When the two-fold degeneracy is lifted then $\alpha=1$. From here on, $N$ refers to the number of distinct transmission eigenvalues (ignoring accidental degeneracies). Furthermore we will assume that the transmission eigenvalues are ordered by magnitude,

$$
T_{1} \leq T_{2} \leq T_{3} \leq \ldots \leq T_{N}
$$

as this results in a number of technical simplifications. The conductance quantum is defined as $G_{0}=\alpha e^{2} / h$.

With these conventions, the transmission eigenvalues determine fundamental transport properties such as the conductance via

$$
G=G_{0} \sum_{n=1}^{N} T_{n}
$$

and the shot-noise power via

$$
P=2 G_{0} e V \sum_{n=1}^{N} T_{n}\left(1-T_{n}\right) .
$$

Here $V$ is the bias voltage, which is assumed to be small.

\section{B. Dyson's circular ensembles}

Random-matrix theory delivers a statistical description of transport by drawing the scattering matrices from ensembles of unitary matrices which obey the constraints of the given universality class. For the three main universality classes with $\beta=1,2$, or 4 , random-matrix theory is based on Dyson's circular ensembles, for which the probability measure is given by the Haar measure of unitary symmetric, unitary, or unitary self-dual matrices, respectively. The joint probability density of transmission eigenvalues then takes the form [1]

$$
P\left(\left\{T_{n}\right\}\right) \propto \prod_{m>n}\left(T_{m}-T_{n}\right)^{\beta} \prod_{l} T_{l}^{-1+\beta / 2} .
$$

The first product in Eq. (5) involves pairs of transmission eigenvalues and favors sequences in which neighboring transmission eigenvalues do not approach each other closely. (As we have ordered the transmission eigenvalues by magnitude, all differences $T_{m}-T_{n}$ are positive.) This suppresses fluctuations in the eigenvalue sequence and ultimately results in conductance fluctuations of the order of a single conductance quantum, which for large $N$ approach the asymptotic value

$$
\operatorname{var} G / G_{0}=\frac{1}{8 \beta} .
$$

For large $N$, the one-point probability density of transmission eigenvalues approaches

$$
P(T)=\frac{1}{\pi \sqrt{T(1-T)}} .
$$

The second product in Eq. (5) induces an asymmetry into this bi-modal distribution, which for large $N$ results in the weak-localization correction

$$
\langle G\rangle-\frac{N}{2} G_{0}=G_{0}\left(\frac{1}{4}-\frac{1}{2 \beta}\right)
$$

of the ensemble-averaged conductance.

An insightful quantity derived from the joint probability density $P\left(\left\{T_{n}\right\}\right)$ is the distribution $P(s)$ of spacings $s=T_{n+1}-T_{n}$ between neighboring transmission eigenvalues. For uncorrelated eigenvalues with average spacing $\bar{s}$ one would expect a Poisson distribution,

$$
P(s)=\bar{s}^{-1} e^{-s / \bar{s}},
$$

while for the circular ensembles and $N \gg 1$, the spacing distribution can be well approximated by the Wigner distributions $[5,6]$,

$$
P(s)=\left\{\begin{array}{ll}
\frac{\pi}{2 \bar{s}^{2}} s \exp \left(-\frac{\pi s^{2}}{4 \bar{s}^{2}}\right) & \beta=1 \\
\frac{32}{\pi^{2} \bar{s}^{3}} s^{2} \exp \left(-\frac{4 s^{2}}{\pi \bar{s}^{2}}\right) & \beta=2 \\
\frac{2^{18}}{3^{6} \pi^{3} \bar{s}^{5}} s^{4} \exp \left(-\frac{64 s^{2}}{9 \pi \bar{s}^{2}}\right) & \beta=4
\end{array} .\right.
$$

Lead-preserving and lead-transposing symmetries entail further constraints on the scattering matrix. The consequences of these constraints for the transmission eigenvalue statistics are explored in the remainder of this paper.

\section{LEAD-PRESERVING SYMMETRIES}

A useful reference point for our subsequent investigation of systems with a lead-transposing symmetry (in Sec. IV) are open systems with a lead-preserving symmetry, to which one can directly apply the standard ideas of desymmetrization. The goal of the present section is to reformulate the resulting random-matrix statistics for the case of a lead-preserving symmetry with $\beta=1$ as a staggered level repulsion, as this will allow us to establish a connection to the case of a lead-transposing symmetry.

\section{A. Constraints on the scattering matrix}

An example of a system with a lead-preserving reflection symmetry is shown in Fig. 1 (c). Figure $2(\mathrm{a}, \mathrm{b})$ shows the desymmetrized version of the system, which is halved at the symmetry line. Dirichlet boundary conditions on the line of symmetry select scattering wave functions with an odd parity, while Neumann boundary 
(a)

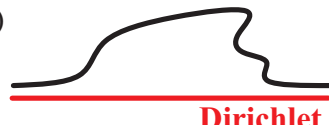

(c)

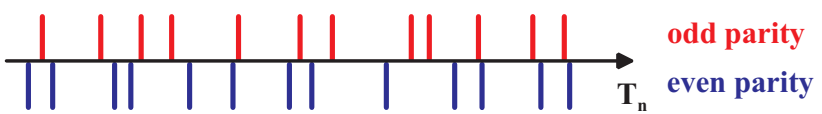

(d)

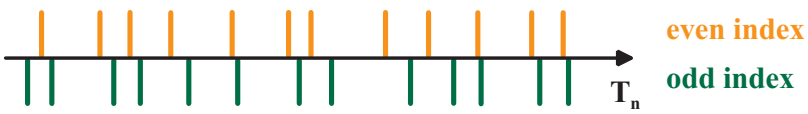

FIG. 2: (Color online) (a,b) Desymmetrization of the quantum billiard with a lead-preserving reflection symmetry, shown in Fig. 1(c). (c) Sketch of the individual transmission eigenvalue sequences of fixed parity. (d) Reorganization as a staggered level sequence, where transmission eigenvalues alternate after ordering by magnitude.

conditions yield even parity. Consequently, the transmission matrix $t$ assumes a block structure where each block corresponds to a given parity. As dictated by the onedimensional transverse-mode quantization in the leads, the block of even parity has dimension $N_{1} \equiv[(N+1) / 2]$, while the block of odd parity has dimension $N_{2} \equiv[N / 2]$ (here [.] denotes the integer part of a number). Hence, both blocks have either the same size (when $N=N_{1}+N_{2}$ is even), or the block with even parity is by one larger than the block with odd parity (when $N$ is odd).

The total transmission-eigenvalue sequence is therefore obtained from a superposition of two sequences of size $N_{1}$ and $N_{2}$ [for illustration see Fig. 2(c)]. In order to fix the way we address the elements of this superposition, we impose the ordering of Eq. (2) and denote by $\mathcal{P}$ the set of all strictly increasing sequences of indices $I_{n} \in\{1,2,3, \ldots, N\}$, where each sequence is of length $N_{1}$. Such sequences are of the form $I=\left(I_{1}, I_{2}, \ldots, I_{N_{1}}\right)$, where $1 \leq I_{1}<I_{2}<I_{3}<\ldots<I_{N_{1}} \leq N$. For each sequence we also define a complementary sequence $\bar{I}=\left(\bar{I}_{1}, \bar{I}_{2}, \bar{I}_{3}, \ldots, \bar{I}_{N_{2}}\right)$, which consists of the indices $1 \leq \bar{I}_{1}<\bar{I}_{2}<\bar{I}_{3}<\ldots<\bar{I}_{N_{2}} \leq N$ not contained in $I$. This partition delivers two ordered subsequences $T_{I_{n}}$ and $T_{\bar{I}_{n}}$.

\section{B. Conventional random-matrix theory}

Within random-matrix theory, the joint probability distribution of the total transmission-eigenvalue sequence is the sum of the corresponding probabilities for each way to distribute the transmission eigenvalues into two sets containing $N_{1}$ and $N_{2}$ eigenvalues. With each sequence obeying the statistics of the appropriate Dyson ensemble one finds with Eq. (5)

$$
\begin{aligned}
P\left(\left\{T_{n}\right\}\right) \propto & \sum_{I \in \mathcal{P}} \prod_{m>n}\left(T_{I_{m}}-T_{I_{n}}\right)^{\beta} \prod_{m>n}\left(T_{\bar{I}_{m}}-T_{\bar{I}_{n}}\right)^{\beta} \\
& \times \prod_{l=1}^{N} T_{l}^{-1+\beta / 2} .
\end{aligned}
$$

For large $N$, the separation into two effectively independent systems with Dirichlet and Neumann boundary conditions naturally results in a doubling of the conductance fluctuations (6) and a doubling of the weaklocalization correction (8). Moreover, level repulsion is only effective for transmission eigenvalues which are part of the same sequence. This modifies the spacing probability density, which can be calculated from the general expression [5]

$$
P(s)=\frac{d^{2}}{d s^{2}} \prod_{i} \int_{0}^{\infty} \int_{0}^{\infty} p_{i}\left(\frac{\rho_{i}}{\rho} s+y+z\right) d y d z
$$

for multiple sequences $i$, where $p_{i}(s)$ is the spacing probability densities of each sequence, while $\frac{\rho_{i}}{\rho}$ is the associated fractional eigenvalue density.

For two sequences following the Wigner distribution (10), the resulting spacing probability densities is $(\bar{s} \equiv 1)$

$$
\begin{gathered}
P_{\beta=1}(s)=\frac{e^{-2 x^{2}}}{2}+\frac{\sqrt{\pi}}{2} x e^{-x^{2}} \mathcal{E}(x), \quad x=\frac{\sqrt{\pi} s}{4}(13) \\
P_{\beta=2}(s)=\frac{6 x^{2} e^{-2 x^{2}}}{\pi}+2 \frac{x-x^{3}}{\sqrt{\pi}} e^{-x^{2}} \mathcal{E}(x)+\frac{\mathcal{E}^{2}(x)}{2}, \\
x=\frac{s}{\sqrt{\pi}} \\
P_{\beta=4}(s)=\frac{x}{3 \sqrt{\pi}}\left(6+4 x^{2}-4 x^{4}\right) e^{-x^{2}} \mathcal{E}(x)+\frac{\mathcal{E}^{2}(x)}{2} \\
+\frac{2 x^{2}}{9 \pi}\left(9+28 x^{2}+8 x^{4}\right) e^{-2 x^{2}}, \quad x=\frac{4 s}{3 \sqrt{\pi}},
\end{gathered}
$$

where $\mathcal{E}(x)=\operatorname{erfc}(x)$ denotes the complementary error function.

\section{C. $\beta=1$ : Reformulation as a staggered eigenvalue sequence}

In most situations encountered in random-matrix theory, the combinatorial sum over partitions involved in the superposition of eigenvalue sequences ([as in Eq. (11)] cannot be performed explicitly. For the specific case $\beta=1$, however, the combinatorial sum over $I$ in Eq. (11) can be carried out (see below), which then yields a closed-form expression

$$
P\left(\left\{T_{n}\right\}\right) \propto \prod_{\substack{m>n, \\ \text { both odd }}}\left(T_{m}-T_{n}\right) \prod_{\substack{m>n, \\ \text { both even }}}\left(T_{m}-T_{n}\right) \prod_{l} \frac{1}{\sqrt{T_{l}}} .
$$


(A similar simplification does not present itself in the cases $\beta=2$ and $\beta=4$.) This result finds its natural statistical interpretation as a staggered superposition of two sequences, which is illustrated in Fig. 2(d). In such a superposition, the transmission eigenvalues in each sequence are not distinguished by the parity of the associated wavefunction under the symmetry operation. Instead, the transmission eigenvalues are ordered by magnitude (irrespective of parity), and one sequence is composed of all odd-indexed transmission eigenvalues (of which there are $N_{1}$ ) while the other sequence is composed of all even-indexed transmission eigenvalues (of which there are $N_{2}$ ). Compared to the original superposition of two independent sequences, this differs by the additional constraint

$$
T_{I_{1}} \leq T_{\bar{I}_{1}} \leq T_{I_{2}} \leq T_{\bar{I}_{2}} \leq T_{I_{3}} \leq T_{\bar{I}_{3}} \ldots
$$

(which is satisfied when all the ordered indices $I_{n}$ are odd while the indices $\bar{I}_{n}$ are all even).

In order to demonstrate the equivalence of Eq. (11) (for $\beta=1$ ) and Eq. (14) we have to show that the levelrepulsion terms are proportional to each other (both expressions share the same product of one-point weights $\prod_{l} T_{l}^{-1 / 2}$, and the proportionality constant is fixed by normalization). We set out to work towards this goal by defining a matrix

$$
M=\left(\begin{array}{cccccccr}
-\mathbf{v}_{1} & \mathbf{v}_{2} & -\mathbf{v}_{3} & \mathbf{v}_{4} & -\mathbf{v}_{5} & \mathbf{v}_{6} & \ldots & (-1)^{N} \mathbf{v}_{N} \\
\mathbf{w}_{1} & \mathbf{w}_{2} & \mathbf{w}_{3} & \mathbf{w}_{4} & \mathbf{w}_{5} & \mathbf{w}_{6} & \ldots & \mathbf{w}_{N}
\end{array}\right),
$$

which is composed of column vectors

$$
\begin{aligned}
& \mathbf{v}_{n}=\left(1, T_{n}, T_{n}^{2}, \ldots, T_{n}^{N_{1}-1}\right)^{T}, \\
& \mathbf{w}_{n}=\left(1, T_{n}, T_{n}^{2}, \ldots, T_{n}^{N_{2}-1}\right)^{T} .
\end{aligned}
$$

The determinant $\operatorname{det} M$ can be evaluated in two different ways. In the first way, we expand it in terms of subdeterminants with $N_{1}$ vectors $\mathbf{v}_{n}$ from the first $N_{1}$ rows and $N_{2}$ vectors $\mathbf{w}_{m}$ from the remaining rows. In other words, we sum over all determinants of the form

$$
\operatorname{det}\left(\begin{array}{ccccccc}
-\mathbf{v}_{1} & \mathbf{v}_{2} & 0 & \mathbf{v}_{4} & 0 & 0 & \cdots \\
0 & 0 & \mathbf{w}_{3} & 0 & \mathbf{w}_{5} & \mathbf{w}_{6} & \cdots
\end{array}\right),
$$

etc., where the indices of the vectors $\mathbf{v}_{I_{n}}$ form an ordered subsequence $I$ and the indices of the vectors $\mathbf{w}_{\bar{I}_{n}}$ are given by the complementary subsequence $\bar{I}$. The alternating signs in front of the vectors $\mathbf{v}_{I_{n}}$ can be pulled out of the determinant at the cost of an overall factor $(-1)^{I_{1}+I_{2}+\ldots+I_{N_{1}}}$. Next, we use permutations of neighboring rows to bring all vectors $\mathbf{v}_{I_{n}}$ to the left (into row $n$ ). This results in an additional sign factor $(-1)^{\left(I_{1}-1\right)+\left(I_{2}-2\right)+\ldots+\left(I_{N_{1}}-N_{1}\right)}$. The determinant of the resulting block matrix factorizes. Overall, this expansion yields

$$
\begin{aligned}
& \operatorname{det} M=(-1)^{N_{1}\left(N_{1}+1\right) / 2} \\
& \times \sum_{I \in \mathcal{P}} \operatorname{det}\left(\mathbf{v}_{I_{1}}, \mathbf{v}_{I_{2}}, \ldots, \mathbf{v}_{I_{N_{1}}}\right) \operatorname{det}\left(\mathbf{w}_{\bar{I}_{1}}, \mathbf{w}_{\bar{I}_{2}}, \ldots, \mathbf{w}_{\bar{I}_{N_{2}}}\right) .
\end{aligned}
$$

Each subdeterminant is of the form of a Vandermonde determinant, and therefore

$$
\begin{aligned}
\operatorname{det} M= & (-1)^{N_{1}\left(N_{1}+1\right) / 2} \\
& \times \sum_{I \in \mathcal{P}} \prod_{m>n}\left(T_{I_{m}}-T_{I_{n}}\right) \prod_{m>n}\left(T_{\bar{I}_{m}}-T_{\bar{I}_{n}}\right) .
\end{aligned}
$$

Secondly, the determinant $\operatorname{det} M$ can be evaluated by adding in Eq. (16) the first $N_{2}$ rows to the last $N_{2}$ rows. This yields

$\operatorname{det} M=\operatorname{det}\left(\begin{array}{ccccccc}-\mathbf{v}_{1} & \mathbf{v}_{2} & -\mathbf{v}_{3} & \mathbf{v}_{4} & -\mathbf{v}_{5} & \mathbf{v}_{6} & \ldots \\ 0 & 2 \mathbf{w}_{2} & 0 & 2 \mathbf{w}_{4} & 0 & 2 \mathbf{w}_{6} & \ldots\end{array}\right)$.

Proceeding again with the evaluation of subdeterminants we are left with a single choice, namely, to select vectors $\mathbf{v}_{n}$ with odd index and vectors $\mathbf{w}_{n}$ with even index. Accounting for all signs and now also factors of two, this results in

$$
\begin{aligned}
\operatorname{det} M= & (-1)^{N_{1}\left(N_{1}+1\right) / 2} 2^{N_{2}} \\
& \times \operatorname{det}\left(\mathbf{v}_{1}, \mathbf{v}_{3}, \mathbf{v}_{5}, \ldots\right) \operatorname{det}\left(\mathbf{w}_{2}, \mathbf{w}_{4}, \mathbf{w}_{6}, \ldots\right) .
\end{aligned}
$$

As this again involves Vandermonde determinants, we find

$$
\begin{aligned}
\operatorname{det} M= & (-1)^{N_{1}\left(N_{1}+1\right) / 2} 2^{N_{2}} \\
& \times \prod_{\substack{m>n, \\
\text { both odd }}}\left(T_{m}-T_{n}\right) \prod_{\substack{m>n, \\
\text { both even }}}\left(T_{m}-T_{n}\right) .
\end{aligned}
$$

The two results Eqs. (21) and (24) deliver the remarkable identity

$$
\begin{aligned}
& \sum_{I \in \mathcal{P}} \prod_{m>n}\left(T_{I_{m}}-T_{I_{n}}\right) \prod_{m>n}\left(T_{\bar{I}_{m}}-T_{\bar{I}_{n}}\right) \\
& =2^{N_{2}} \prod_{\substack{m>n, \\
\text { both odd }}}\left(T_{m}-T_{n}\right) \prod_{\substack{m>n, \\
\text { both even }}}\left(T_{m}-T_{n}\right),
\end{aligned}
$$

which shows that the level-repulsion term in Eq. (11) is indeed proportional to the level-repulsion term in Eq. (14). As already mentioned, the one-point product $\prod_{l} T_{l}^{-1 / 2}$ in both expressions is identical, and the proportionality constant is fixed by normalization. It follows that for $\beta=1$, the independent superposition of two transmission-eigenvalue sequences with $N_{1}$ and $N_{2}$ levels (with $N_{1}$ and $N_{2}$ constrained to differ at most by one) is identical to a staggered superposition of two transmission-eigenvalue sequences with $N_{1}$ and $N_{2}$ levels, which are correlated by the ordering requirement (15).

\section{LEAD-TRANSPOSING SYMMETRIES}

Systems with a lead-transposing symmetry require a separate treatment since the symmetry operation only commutes with the Hamiltonian, but not with the current operator (which changes its sign). In the presence 
(a)

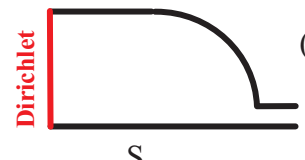

(b)

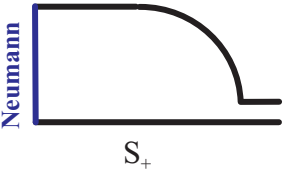

(c)

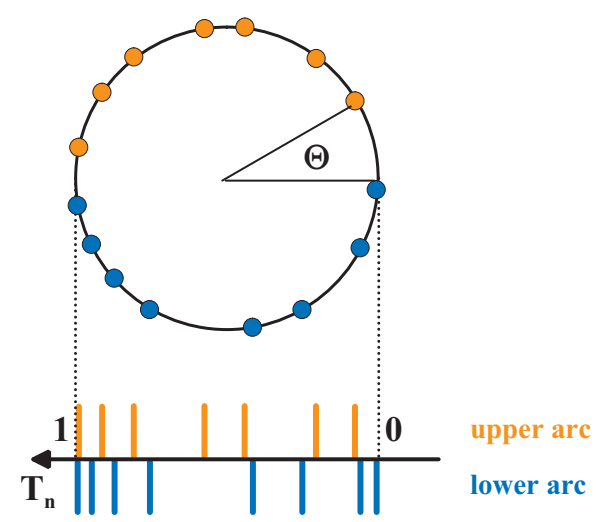

FIG. 3: (Color online) (a,b) Desymmetrization of the quantum billiard with a lead-transposing reflection symmetry, shown in Fig. 1(e). (c,d) Eigenphases $\Theta_{n}$ of the matrix $Q$ on the unit circle, and their projection Eq. (28) which delivers the transmission eigenvalues.

of an applied bias, the symmetry operation exchanges the electronic source and drain reservoirs. An obvious symptom of this complication is the fact that the desymmetrized system only possesses a single lead (see Fig. 3). Mathematically, the transmission matrix does not assume a block structure but remains full. We will first adapt the concept of desymmetrization to derive the constraints of the scattering matrix, and then turn to the joint probability density of the transmission eigenvalues in random-matrix theory. Just as in the previous section, we then focus on the orthogonal symmetry class $(\beta=1)$ and derive a closed expression for the joint probability density, which again assumes the form of a staggered level repulsion.

\section{A. Constraints on the scattering matrix}

The presence of a lead-transposing symmetry immediately results in the constraint $r=r^{\prime}, t=t^{\prime}$ (when timereversal symmetry is broken by a magnetic field, this can be achieved by an inversion symmetry but not by a reflection symmetry). In order to further exploit the consequences of the symmetry, let us inspect a time-reversal symmetric system with a reflection symmetry, as shown in Fig. 1(e). As shown in Fig. 3(a,b), the desymmetrized versions are cut at the symmetry line, where they are equipped with Dirichlet or Neumann boundary conditions for wavefunctions of odd $(-)$ or even parity $(+)$, respectively. Such wave functions are readily constructed starting from the original system when one chooses incoming amplitudes of the form $\mathbf{a}^{(R)}= \pm \mathbf{a}^{(L)}$. The outgoing amplitudes are then given by $\mathbf{b}^{(L)}=(r \pm t) \mathbf{a}^{(L)}$. Con- sequently, the scattering matrices of the desymmetrized systems are given by

$$
S_{ \pm}=r \pm t
$$

The desymmetrized systems only possess a single opening. In order to revert to the scattering matrix of the original system we invert Eq. (26). The transport in the original system is therefore described by the transmission matrix $t=\frac{1}{2}\left(S_{+}-S_{-}\right)$, which gives

$$
t t^{\dagger}=\frac{1}{4}\left(2-S_{+} S_{-}^{\dagger}-S_{-} S_{+}^{\dagger}\right)
$$

The properties of this matrix - and especially, of its eigenvalues $T_{n}$ - are not separable and depend on the interplay of both desymmetrized variants.

\section{B. Conventional random-matrix theory}

Random-matrix ensembles for systems with leadtransposing symmetry can be obtained by assuming that the scattering matrices $S_{+}$and $S_{-}$of the desymmetrized variants are statistically independent realizations of the appropriate standard circular ensemble. The resulting ensembles are identical to those introduced by Baranger and Mello [9], who based their considerations on a maximal-entropy principle.

Earlier works have addressed isolated aspects of these ensembles, but not the complete transmission-eigenvalue statistics. For instance, it has been observed that a leadtransposing symmetry increases the conductance fluctuations [8, 11] but eliminates the weak-localization correction [11]. For large $N$, the conductance fluctuations double, just as is the case for lead-preserving symmetries. We now provide a complete explanation of these observations on the basis of the joint probability density of the transmission eigenvalues.

The starting point of these considerations is the relation

$$
T_{n}=\sin ^{2}\left(\Theta_{n} / 2\right)=\frac{1}{2}\left(1-\cos \Theta_{n}\right)
$$

between the transmission eigenvalues $T_{n}$ and the eigenphases $\Theta_{n}$ of the unitary matrix $Q \equiv S_{+} S_{-}^{\dagger}$, which follows from Eq. (27). As illustrated in Fig. 3(c,d), the statistics of transmission eigenvalues is hence directly imposed by the statistics of the real parts $\cos \Theta_{n}$ of the unimodular eigenvalues $e^{i \Theta_{n}}$ of $Q$.

In random-matrix theory, the eigenphases $\Theta_{n}$ follow the statistics of the associated circular ensemble. This is evident for the unitary ensemble $(\beta=2)$, which is invariant under the multiplication of an arbitrary fixed matrix (it hence suffices, e.g., to assume that $S_{+}$is random while $S_{-}$is fixed, or vice versa). In the orthogonal case $(\beta=1)$, the unitary transformation $Q^{\prime}=S_{-}^{-1 / 2} Q S_{-}^{1 / 2}=$ $S_{-}^{-1 / 2} S_{+} S_{-}^{-1 / 2}$ results in a symmetric matrix with identical eigenvalues. Their circular statistics then follows from 
the fact that the circular orthogonal ensemble is invariant under the symmetric involution with any fixed symmetric matrix (here, $S_{-}^{-1 / 2}$ ). The same transformation also succeeds in the case of self-dual matrices $(\beta=4)$.

Because of the uniform distribution of eigenphases in the circular ensemble [5], the one-point probability density $P\left(T_{n}\right)$ is given by Eq. (7) for any finite $N$ (i.e., not only in the limit $N \rightarrow \infty$ ) [11]. The joint probability density of the eigenphases $\Theta_{n}$ is given by [5]

$$
P_{\Theta}\left(\left\{\Theta_{n}\right\}\right) \propto \prod_{m>n}\left[\sigma_{m} \sin \frac{\Theta_{m}-\Theta_{n}}{2}\right]^{\beta} .
$$

Here we ordered the eigenphases by their moduli,

$$
0 \leq\left|\Theta_{1}\right| \leq\left|\Theta_{2}\right| \leq\left|\Theta_{3}\right| \leq \ldots \leq\left|\Theta_{N}\right| \leq \pi,
$$

and denoted $\sigma_{n}=\operatorname{sgn} \Theta_{n}$. Since Eq. (28) does not discriminate the sign of $\Theta_{n}$ we proceed to the distribution of the moduli $\theta_{n}=\left|\Theta_{n}\right|$,

$$
P_{\theta}\left(\left\{\theta_{n}\right\}\right)=\sum_{\left\{\sigma_{n}\right\}} P_{\Theta}\left(\left\{\sigma_{n} \theta_{n}\right\}\right) .
$$

With the help of the relations

$$
\sin \left(\theta_{n} / 2\right)=\sqrt{T_{n}}, \quad \cos \left(\theta_{n} / 2\right)=\sqrt{1-T_{n}},
$$

and also accounting for the Jacobian

$$
\frac{d \theta_{n}}{d T_{n}}=\frac{1}{\sqrt{T_{n}\left(1-T_{n}\right)}}
$$

this yields the joint probability density [11]

$$
\begin{aligned}
& P\left(\left\{T_{n}\right\}\right) \propto \prod_{l} \frac{1}{\sqrt{T_{l}\left(1-T_{l}\right)}} \\
& \times \sum_{\left\{\sigma_{n}\right\}} \prod_{m>n}\left[\sqrt{T_{n}\left(1-T_{m}\right)}-\sigma_{m} \sigma_{n} \sqrt{T_{m}\left(1-T_{n}\right)}\right]^{\beta} .
\end{aligned}
$$

This expression is symmetric under the replacement $T_{n} \rightarrow 1-T_{n}$, which explains the absence of weaklocalization corrections to the conductance. Moreover, transmission eigenvalues do not repel each other when $\sigma_{n}=-\sigma_{m}$, i.e., when the underlying eigenphases $\Theta_{n}$ lie on the opposite (upper and lower) arcs of the unit circle [see again Fig. 3(c)]. As the sets of eigenphases on both arcs is only weakly cross-correlated, this explains the doubling of the conductance fluctuations for large $N$.

\section{Staggered level repulsion for $\beta=1$}

While the general conclusions of the previous section can be drawn for any $\beta$, it should be noted that Eq. (34) still involves a combinatorial sum, and hence is similar in status as expression (11) for systems with a leadpreserving symmetry. We now show that a much more detailed insight is possible for the orthogonal symmetry class $(\beta=1)$, where the combinatorial sum in Eq. (34) can be carried out explicitly (see below). The resulting statistics again assume the form of a staggered level repulsion, but are not identical to Eq. (14) (which was derived from the superposition of two independent level sequences): For $N$ an odd integer, we find

$$
\begin{aligned}
P\left(\left\{T_{n}\right\}\right) & \propto \prod_{m>n, \text { both odd }}\left(T_{m}-T_{n}\right) \prod_{l \text { odd }} \frac{1}{\sqrt{T_{l}\left(1-T_{l}\right)}} \\
& \times \prod_{m>n, \text { both even }}\left(T_{m}-T_{n}\right),
\end{aligned}
$$

while for even $N$

$$
\begin{aligned}
P\left(\left\{T_{n}\right\}\right) & \propto \prod_{m>n, \text { both odd }}\left(T_{m}-T_{n}\right) \prod_{l \text { odd }} \frac{1}{\sqrt{T_{l}}} \\
& \times \prod_{m>n, \text { both even }}\left(T_{m}-T_{n}\right) \prod_{l \text { even }} \frac{1}{\sqrt{1-T_{l}}} .
\end{aligned}
$$

Similar to Eq. (14), the joint probability density again separates into two factors, each involving only every second eigenvalue. In particular, neighboring levels are not prohibited to approach each other closely, and statistical fluctuations of observables are enhanced, as has been earlier observed for the conductance and the Fano factor [8-11]. The correlation between the two level sequences is again imposed only indirectly by the requirement that the sequences are staggered. This ordering requirement is independent of the parity of the wave function - indeed, in the present case, parity is not well defined as the transmission eigenvalues arise from the combined properties of $S_{+}$and $S_{-}$.

In order to derive Eq. (35), let us first inspect Eq. (29). Because of the ordering (30), each factor $\sigma_{m}$ appears $m-$ 1 times, and therefore

$$
P_{\Theta}\left(\left\{\Theta_{n}\right\}\right) \propto \prod_{l \text { even }} \sigma_{l} \prod_{m>n} \sin \frac{\Theta_{m}-\Theta_{n}}{2} .
$$

We next pass over to the joint distribution of moduli (31). In order to evaluate the combinatorial sum over the $\sigma_{n}$ we express the factor of sine functions in Eq. (36) as a Vandermonde determinant,

$$
\prod_{m>n} \sin \frac{\Theta_{m}-\Theta_{n}}{2}=(-i)^{N(N-1) / 2} \operatorname{det} B\left(\left\{\sigma_{n} \theta_{n}\right\}\right)
$$

where $B_{m l}\left(\left\{\Theta_{n}\right\}\right)=\exp \left(i \Theta_{m} l\right), m=1,2,3, \ldots, N$, while the index $l$ runs in integer steps from $-(N-1) / 2$ to $(N-1) / 2$. The multilinearity of the determinant then yields

$$
P_{\theta}\left(\left\{\theta_{n}\right\}\right) \propto(-i / 2)^{N(N-1) / 2} \operatorname{det} C,
$$

where $C_{m l}=2 \cos \left(\theta_{m} l\right)$ for odd $m$ and $C_{m l}=2 i \sin \left(\theta_{m} l\right)$ for even $m$. 
For every $l>0$ we now add the $l$ th column in $C$ to the -lth column, which cancels all sine terms in the latter columns. The determinant $\operatorname{det} C=\operatorname{det} D \operatorname{det} E$ then factorizes, where $D_{m l}=\cos \theta_{m} l, m$ odd, and $E_{m l}=\sin \theta_{m} l$, $m$ even. If $N$ is even, the index $l$ is now restricted to $l=1 / 2,3 / 2, \ldots,(N-1) / 2$. For odd $N$, this index is restricted to $l=0,1,2, \ldots,(N-1) / 2$ for the matrix $D$, and to $l=1,2, \ldots,(N-1) / 2$ for the matrix $E$.

For odd $N$ we can write $D_{m l}$ as a polynomial of degree $l$ in $\cos \theta_{m}$, and $E_{m l}$ as $\sin \theta_{m}$ times a polynomial of degree $l-1$ in $\cos \theta_{m}$. We only need to keep the highest monomial, as the other terms are linear combinations of the rows of lower index $l$. This leaves us again with Vandermonde determinants,

$$
\begin{aligned}
& \operatorname{det} D \propto \prod_{m>n, \text { both odd }}\left(\cos \theta_{n}-\cos \theta_{m}\right) \\
& \operatorname{det} E \propto \prod_{l \text { even }} \sin \theta_{l} \prod_{m>n, \text { both even }}\left(\cos \theta_{n}-\cos \theta_{m}\right) .
\end{aligned}
$$

For even $N$, the index $l$ is half-integer, and the elements of $D$ can now be written as $\cos \left(\theta_{m} / 2\right)$ times a polynomial in $\cos \left(\theta_{m}\right)$, while those of $E$ can be written as $\sin \left(\theta_{m} / 2\right)$ times such a polynomial. This yields

$$
\begin{aligned}
& \operatorname{det} D \propto \prod_{l \text { odd }} \cos \left(\theta_{l} / 2\right) \prod_{m>n, \text { both odd }}\left(\cos \theta_{n}-\cos \theta_{m}\right), \\
& \operatorname{det} E \propto \prod_{l \text { even }} \sin \left(\theta_{l} / 2\right) \prod_{m>n, \text { both even }}\left(\cos \theta_{n}-\cos \theta_{m}\right) .
\end{aligned}
$$

The joint probability density (35) follows by transforming from $\theta_{n}$ to $T_{n}$, where the Jacobian is given by Eq. (33), while the factors in the expressions for $D$ and $E$ can be rewritten with the help of Eq. (32) and the relation $\cos \theta_{n}-\cos \theta_{m}=2\left(T_{m}-T_{n}\right)$.

\section{Large- $N$ asymptotics}

It is natural to ask whether the similarity of Eq. (35) to Eq. (14) indicates a possible interpretation as a superposition of two independent level sequences [from which Eq. (14) was derived]. In Eq. (35), however, this interpretation is prevented by the different one-point weight terms associated to the even and odd indexed eigenvalues. A symptom of this difference is the fact that Eq. (14) implies finite- $N$ weak-localization corrections to the conductance, while Eq. (35) delivers the absence of such corrections, in agreement with the general conclusions in Sec. IV B. Hence, the statistics of systems with a leadtransposing and a lead-preserving symmetry (with $\beta=1$ ) only find a common ground when both are interpreted as a staggered level sequence.

For the case of a lead-preserving symmetry, the framework of superpositions of independent level sequences of course provides a powerful tool for the derivation of low-point correlation functions and local statistics [such as the two-point correlation function, or the level spacing distribution (13)]. We now argue that in the limit $N \rightarrow \infty$, this framework can also be adopted for systems with a lead-transposing symmetry.

In this limit, the transmission eigenvalues form a quasicontinuum, and the asymptotical statistics follow from the formal analogy to the statistics of coordinates of a dense set of parallel line charges in one dimension (the Coulomb gas), which exhibit a logarithmic repulsion $[1,5]$. In leading order, the weight terms enter the analysis of the statistical fluctuations only via the onepoint function $P(T)$ : For fixed index $n$, the transmission eigenvalue $T_{n}$ are confined to a small neighborhood around a nominal equilibrium position $\bar{T}_{n}$, which is given by the implicit equation $n-1 / 2=N \int_{0}^{\bar{T}_{n}} P(T) d T$. Subsequently, the weight terms can be approximated by a constant (with all the $T_{n}$ fixed to $\bar{T}_{n}$ ), while the fluctuations are exclusively governed by the level-repulsion factors of the joint probability distribution. As the levelrepulsion factors are identical in Eqs. (14) and (35) one concludes that the local statistics in both ensembles become indistinguishable in the limit of $N \rightarrow \infty$.

We therefore obtain the following remarkable result of purely statistical origin: For a lead-transposing symmetry, as $N$ is sent to infinity the local statistics (embodied in low-point correlation functions) converges to that of a superposition of two independent level sequences. This is the case even though a classification of transmission eigenvalues by parity is not possible. In particular, we arrive at the prediction that in this limit, the level-spacing distribution is well approximated by Eq. (13).

\section{NUMERICAL INVESTIGATIONS}

For the three standard Dyson ensembles of randommatrix theory, the joint probability density (5) manifests the celebrated repulsion between neighboring eigenvalues, since the probability to find two closely spaced adjacent eigenvalues is suppressed as $\left(T_{n+1}-T_{n}\right)^{\beta}$. In contrast, the joint densities (14) and (35) (both derived for $\beta=1$ ) describe sequences of reduced stiffness, where only every second level is subject to mutual level repulsion. As argued before, as long as $N$ takes on moderate values, the latter joint densities imply quantitative differences in the transmission eigenvalue statistics for lead-preserving and lead-transposing symmetries, while for large $N$ these statistics should converge onto each other.

In this section we illustrate the differences and similarities between these scenarios for all three main symmetry classes $(\beta=1,2,4)$ via numerical sampling of the random-matrix ensembles, and also compare to realistic model systems. For convenient characterization of the eigenvalue repulsion we employ the nearest-neighbor spacing distribution $P(s)$, as well as spacing distributions to more distant neighbors. As we will see, the local statistics of systems with a lead-transposing symmetry actually show a much weaker $N$ dependence than for 


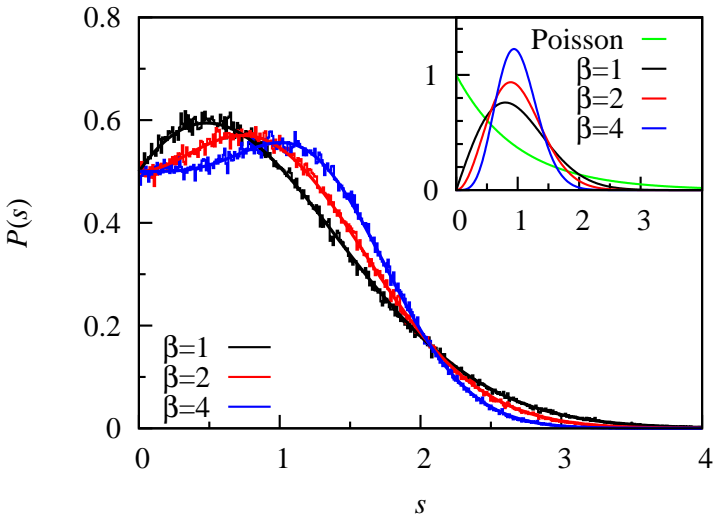

FIG. 4: (Color online) Probability density $P(s)$ of transmission-eigenvalue spacings for systems with a leadtransposing symmetry, obtained from $10^{4}$ random matrices with $N=50$. Smooth curves: Spacing probability density (13) for superpositions of eigenvalues of two independent sequences from the standard circular ensembles. The inset shows the Wigner distributions (10) from standard randommatrix theory and the Poisson distribution (9) for uncorrelated eigenvalues.

systems with a lead-preserving symmetry. This feature could be anticipated by (but also goes beyond) the absence of weak localization corrections in the one-point function (discussed in Sec. IV B).

\section{A. Random-matrix theory}

We start with the characterization of the level statistics within the various random-matrix ensembles. Let us first consider the case of a lead-transposing symmetry with a relatively large number of transport channels, for which we expect that the local statistics is close to that of a superposition of two independent level sequences. Starting point of the numerical computations is Eq. (27), where the matrix $Q=S_{+} S_{-}^{\dagger}$ is drawn from the appropriate Dyson ensemble. In order to obtain the nearest-neighbor spacing distribution $P(s)$, we unfold the eigenvalue sequences to a mean local spacing $\bar{s} \equiv 1[5,6]$. Figure 4 shows the resulting spacing distributions for $N=50$. For this large number of open channels we find that the numerical histograms indeed match the predictions from the superposition of two independent level sequences [solid curves; see Eq. (13)].

For comparison, the inset in Fig. 4 shows the standard Wigner distributions (10), as well as the Poisson distribution (9). In the Poisson distribution the eigenvalue spacing density is maximal at $s=0$; for larger $s$ the probability density decreases monotonically. For the Wigner distributions the most likely eigenvalue spacing occurs at a finite value of $s$; for $s \rightarrow 0$, the distributions decay algebraically $\propto s^{\beta}$, while for $s \rightarrow \infty$ they decay as a Gaussian. The distributions in the main panel combine

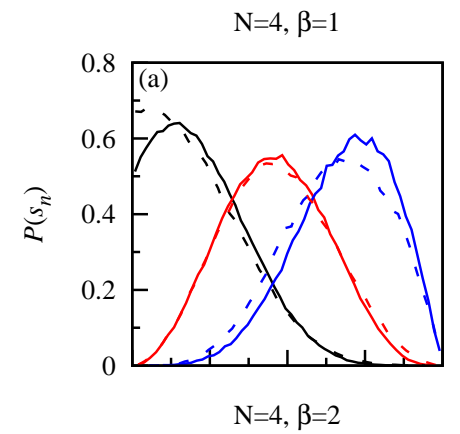

$\mathrm{N}=100, \beta=1$
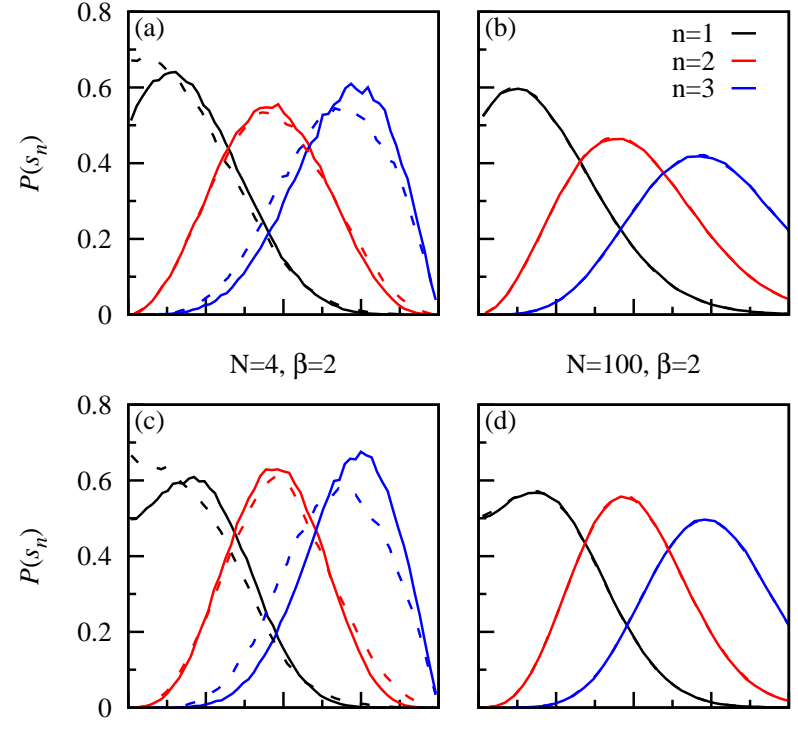

$\mathrm{N}=100, \beta=4$
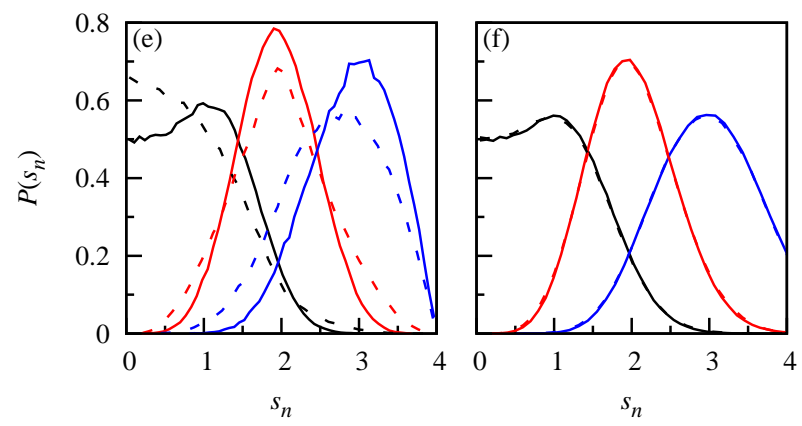

FIG. 5: (Color online) Probability densities of spacings $s_{n}$ to the first, second and third neighboring transmission eigenvalue for the random-matrix ensembles of systems with a leadtransposing symmetry (solid curves) or a lead-preserving symmetry (dashed curves). In the left panels the number of transport channels $N=4$, while in the right panels $N=100$. Top panels: orthogonal symmetry class $(\beta=1)$. Middle panels: unitary symmetry class $(\beta=2)$. Bottom panels: symplectic symmetry class $(\beta=4)$. For each ensemble, the results represent a sample of $10^{4}$ realizations.

the partial absence of level repulsion for small $s$ [with $P(s=0)=1 / 2]$ with the Gaussian decay of the Wigner distributions for large $s$.

For large $N$, virtually identical results are obtained for the conventional case of a lead-preserving symmetry. This is demonstrated in detail in Fig. 5, which also shows the spacing distributions to the second and third-nearest neighbor. Here, solid curves are for a lead-transposing symmetry, and dashed curves are for a lead-preserving symmetry (corresponding to a superposition of independent level sequences from the appropriate Dyson ensemble). For $N=100$ (right panels), dashed and solid curves lie on top of each other and are practically indistinguishable. This clearly supports the convergence of the local 


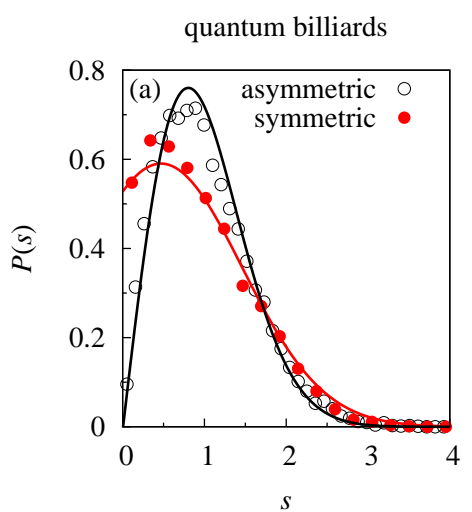

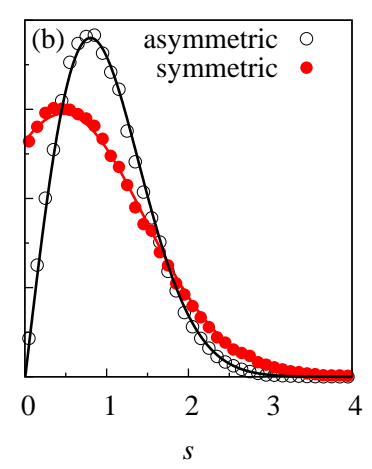

open kicked rotators

FIG. 6: (Color online) (a) Nearest-neighbor spacing distribution $P(s)$ for the lead-asymmetric stadium billiard of Fig. 1 (b), averaged over energies in the range $N=5-14$, and the lead-transposing symmetric stadium billiard of Fig. 1(e), with $N=5,6$. (b) The same for open quantum kicked rotators with $N=12$. In both panels, the solid curves show the Wigner distribution (10) with $\beta=1$ and the prediction of random-matrix theory for systems with a lead-transposing symmetry [which can be safely approximated by Eq. (13a)].

statistics of both cases for large $N$.

The left panels in Fig. 5 show the level-spacing distributions for $N=4$. In this case, the results for a lead-transposing symmetry are distinctively different from those for a lead-preserving symmetry. Interestingly, the nearest-neighbor spacing distribution for a lead-transposing symmetry is very similar for small and large $N$; the distribution for $N=4$ is already well approximated by Eq. (13). In comparison, the nearestneighbor spacing distribution for a lead-preserving symmetry shows a much stronger $N$-dependence.

\section{B. Comparison to model systems}

In order to validate that realistic quantum systems can indeed be described by random matrix theory (on which all previous considerations are based), we compare our predictions with numerical results for such systems. In particular, we present results of numerical computations for quantum billiards, which model a lateral quantum dot, and for the open kicked rotator, which is based on an efficient quantum map. We focus on systems in the orthogonal symmetry class $(\beta=1)$ and contrast systems with a lead-transposing symmetry to systems without any spatial symmetry.

The quantum billiards are derived from the stadium geometry, with leads positioned to either break or conserve the reflection symmetry about the vertical center line [see Figs. 1(b,e)]. The computations are performed using a modular recursive Green's function method [12, 13], with energies that permit $5 \leq N \leq 14$ open channels in each of the two leads. As shown in Fig. 6(a), the eigenvalue spacing distribution agrees well with the predictions of random-matrix theory, both in presence and in absence of a lead-transposing symmetry.

The open quantum kicked rotator [14-16] is defined by the scattering matrix

$$
S=P\left[e^{-i \varepsilon}-F\left(1-P^{T} P\right)\right]^{-1} F P^{T},
$$

where $\varepsilon$ is the quasi-energy,

$$
F_{n m}=(i M)^{-1 / 2} e^{\frac{i \pi}{M}(m-n)^{2}-\frac{i M K}{4 \pi}\left(\cos \frac{2 \pi n}{M}+\cos \frac{2 \pi m}{M}\right)}
$$

is the $M \times M$-dimensional Floquet operator of the kicked rotator, and $P$ is an $2 N \times M$-dimensional matrix which projects the internal Hilbert space onto the openings. We assume that $M$ is even and $M \gg N$. The reflection symmetry of the closed system is manifested in the symmetry $F_{n m}=F_{M-n, M-m}$, and the lead-transposing symmetry of the open system is present when in addition $P_{n m}=P_{2 N-n, M-m}$.

Figure 6(b) shows the spacing distributions obtained for kicked rotators with symmetrical and asymmetrical lead placement and $N=12$. The data represents 6600 realizations which are generated by varying the quasienergy $\varepsilon \in[0,2 \pi)$, the kicking strength $K \in[10,15]$, and the internal dimension $M \in[498,502]$. Again, we find good agreement with random-matrix theory, including the reduced eigenvalue repulsion in the lead-symmetric case.

The results in this section reveal clear signatures of staggered level repulsion in realistic systems with a leadtransposing symmetry (and $\beta=1$ ). It is worth emphasizing that the applicability of this statistical concept [embodied in the random-matrix results Eq. (35)] does not rely on any pre- or postprocessing or -selection of the transmission eigenvalues in the model systems (as there is no intrinsic property of the transmission eigenvalues or their associated scattering wave functions - such as a parity - that could be used to divide these eigenvalues into two sets).

\section{SUMMARY AND CONCLUSIONS}

We analyzed the transport in open systems with a leadtransposing or a lead-preserving symmetry via the complete joint probability density of transmission eigenvalues, obtained in random-matrix theory.

For a lead-preserving symmetry, the standard concept of desymmetrization reduces the problem to the investigation of independent non-symmetric variants of the system. For a lead-transposing symmetry, however, the transport characteristics only arise as a collective property of the symmetry-reduced variants of the system. We still found that both types of symmetry result in a similar reduction of level repulsion, so that transmission eigenvalues can approach each other closely. For a large number of transport channels $N$, the local eigenvalue statistics for both types of symmetry indeed become indistinguishable. 
Our main analytical results concern a detailed explanation of these features for systems which also exhibit time-reversal and spin-rotation invariance (the orthogonal symmetry class, with symmetry index $\beta=1$ ). In this case, the transmission eigenvalue statistics of systems with a lead-transposing or lead-preserving symmetry find a common natural interpretation as a staggered superposition of two independent level sequences. In such a superposition the eigenvalues alternate between the sequences when they are ordered by magnitude. The joint probability densities for the two types of symmetry only differ in one-point weight factors. For lead-preserving symmetries these weight factor incorporate $1 / N$ corrections for quantities such as the ensemble-averaged conductance, while these corrections are absent for a leadtransposing symmetry. This results in differences of the local eigenvalue statistics when $N$ is small, but becomes insignificant when $N$ is large.
While we concentrated on systems with discrete spatial symmetries, our results can also be applied for discrete symmetries of different origin (e.g., arising from internal degrees of freedom) that yield equivalent constraints on the scattering matrix.

\section{Acknowledgments}

We gratefully acknowledge assistance with the billiard computations by Florian Aigner, as well as useful discussions with Eugene Bogomolny, Piet Brouwer, Victor Gopar, Jon Keating, and Martin Zirnbauer. This work was supported by the European Commission, Marie Curie Excellence Grant MEXT-CT-2005-023778. S.R. wishes to thank the Max-Kade foundation and the W.M. Keck foundation for support.
[1] C. W. J. Beenakker, Rev. Mod. Phys. 69, 731 (1997).

[2] F. J. Dyson, J. Math. Phys. 3, 140 (1962).

[3] A. Altland and M. R. Zirnbauer, Phys. Rev. B 55, 1142 (1997).

[4] Y. M. Blanter and M. Büttiker, Phys. Rep. 336, 1 (2000).

[5] M. L. Mehta, Random Matrices, 3rd ed. (Elsevier, 2004).

[6] F. Haake, Quantum signatures of Chaos, 2nd ed. (Springer, Berlin, 2001).

[7] J. P. Keating and J. M. Robbins, J. Phys. A 30, L177 (1997).

[8] V. Gopar, M. Martínez, P. Mello, and H. Baranger, J. Phys. A 29, 881 (1996).

[9] H. U. Baranger and P. A. Mello, Phys. Rev. B 54, R14297 (1996).

[10] H. Schanze, H.-J. Stöckmann, M. Martínez-Mares, and
C. H. Lewenkopf, Phys. Rev. E 71, 016223 (2005).

[11] V. A. Gopar, S. Rotter, and H. Schomerus, Phys. Rev. B 73, 165308 (2006).

[12] S. Rotter, J.-Z. Tang, L. Wirtz, J. Trost, and J. Burgdörfer, Phys. Rev. B 62, 1950 (2000).

[13] S. Rotter, F. Aigner, and J. Burgdörfer, Phys. Rev. B 75, 125312 (2007).

[14] J. Tworzydło, A. Tajic, H. Schomerus, and C. W. J. Beenakker, Phys. Rev. B 68, 115313 (2003)

[15] P. Jacquod, H. Schomerus, and C. W. J. Beenakker, Phys. Rev. Lett. 90, 207004 (2003).

[16] A. Ossipov, T. Kottos, and T. Geisel, Europhys. Lett. 62, 719 (2003). 